nales. Dégénérescence du thymus et du corps thyroïde. Reins normaux quand la mort est précoce, mais seulement dans ce cas.

La pathogénie en est mal connue. Il ne s'agit pas d'avitaminose, car la cuisson diminue ce pouvoir toxique du soja.

Comme la cystine et la méthionine améliorent l'état du rat nourri au soja cru, il est probable que, dans ce cas, il s'agit d'un trouble dans le métabolisme et dans l'équilibre des acides aminés.

\title{
LE TEST de LA PHOSPHATASE APPLIQUé A LA RECHERCHE DE LA PASTEURISATION DES LAITS DE FROMAGERIE
}

par

\author{
A. CAMUS et R. ALIFAX \\ Station centrale de Technologie agricole
}

Le rôle prépondérant joué par la flore superficielle dans la maturation des fromages et l'existence de phosphatases d'origine fongique et bactérienne nous ont conduits à rechercher l'influence des agents microbiens de la maturation sur l'épreuve de la phosphatase appliquée aux fromages.

Dans une communication précédente [1], nous avions insisté sur le fait que la phosphatase qui prend naissance dans la croûte d'un fromage semblait diffuser lentement dans la masse de celui-ci et pouvait troubler les résultats de la recherche de la pasteurisation des laits mis en œuvre pour sa fabrication.

Il nous a paru intéressant de déterminer la vitesse de cette diffusion afin d'établir les délais dans lesquels l'épreuve garde quelque valeur.

Les essais ont porté sur des fromages à pâte molle, à croûte moisie et sur des fromages à pâte pressée cuite.

\section{Essais effectués sur fromages à pâte molle à croûte moisie Camembert.}

Une série de fromages a été fabriquée à partir du lait pasteurisé d'une même cuve et ensuite traitée normalement. Le test de la phosphatase leur a été appliqué à divers stades de la fabrication. Les prélèvements ont été faits, avec toutes les précautions nécessaires pour éviter les apports de la diastase vers le centre, à la surface, à $5 \mathrm{~mm}$. de la surface, au centre du fromage. Nous avons utilisé pour le dosage de la phosphatase la méthode de Sanders et Sager [2].

Les résultats, exprimés en millièmes de milligrammes de phénol 
libéré du phénylphosphate par la phosphatase sont consignés au Tableau I et sur le graphique I.

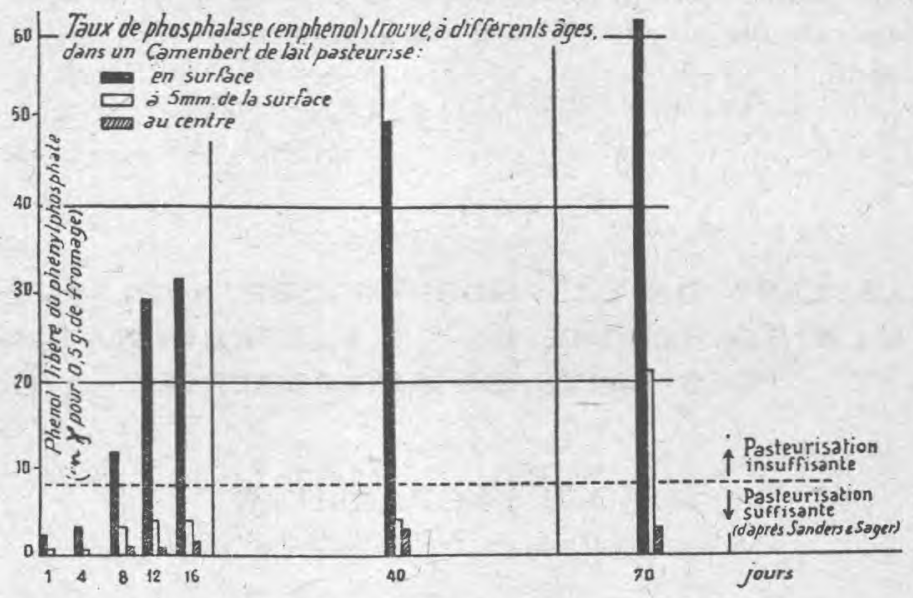

TABLEAU I

PHÉNOL LIBÉRE DU PHÉNYLPHOSPHATE (EN $\gamma$ )

\begin{tabular}{|c|c|c|c|c|c|c|c|}
\hline 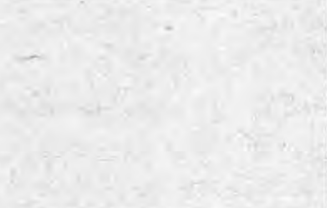 & $\begin{array}{c}\text { 1er jour } \\
\text { après } \\
\text { mou- } \\
\text { lage }\end{array}$ & $\begin{array}{c}4^{e} \text { jour } \\
\text { après } \\
\text { salage }\end{array}$ & $\begin{array}{c}\mathrm{e}^{\mathrm{e}} \text { jour } \\
\text { appa- } \\
\text { rition } \\
\mathrm{du} \\
\text { Peni- } \\
\text { cillium }\end{array}$ & $\begin{array}{c}12^{\mathrm{e}} \\
\text { jour }\end{array}$ & $\begin{array}{l}16^{\mathrm{e}} \\
\text { jour }\end{array}$ & $\begin{array}{c}40^{\mathrm{e}} \\
\text { jour }\end{array}$ & $\begin{array}{l}70^{\circ} \\
\text { jour }\end{array}$ \\
\hline En surface $\ldots \ldots \ldots$ & 2 & 3 & 12 & 30 & 32 & 50 & 62 \\
\hline A $5 \mathrm{~mm}$. de la surface. & 0 & 0 & 3 & 4 & 4 & 4 & 21 \\
\hline Au centre $\ldots \ldots \ldots \ldots$ & 0 & 0 & $\mathbf{l}$ & 1 & 2 & 3 & 3 \\
\hline
\end{tabular}

Ils indiquent que dans les délais normaux de maturation des fromages du type Camembert (20 à 30 jours), la phosphatase ne semble pas apparaître assez rapidement dans le centre du fromage pour gêner la recherche de l'efficacité de la pasteurisation du lait mis en œurre.

\section{Carré de l'Est}

Ccpendant, l'élévation brusque de la quantité de phénol libéré dans la couche située à $5 \mathrm{~mm}$. de la croûte quand la pâte du fromage se solubilise devait nous inciter à faire un essai analogue sur le fromage "Carré de l'Est ».

Ce fromage à croûte moisie diffère du Camembert par sa forme il est moins épais - et par sa fabrication : il provient d'un caillé 
divisé. Sa maturation en profondeur est par suite plus rapide. Le test reste-t-il valable pour cette variété de fromage?

Le tableau II et le graphique II montrent, en effet, une variation beaucoup plus rapide du taux de phosphatase à $5 \mathrm{~mm}$. de la surface,

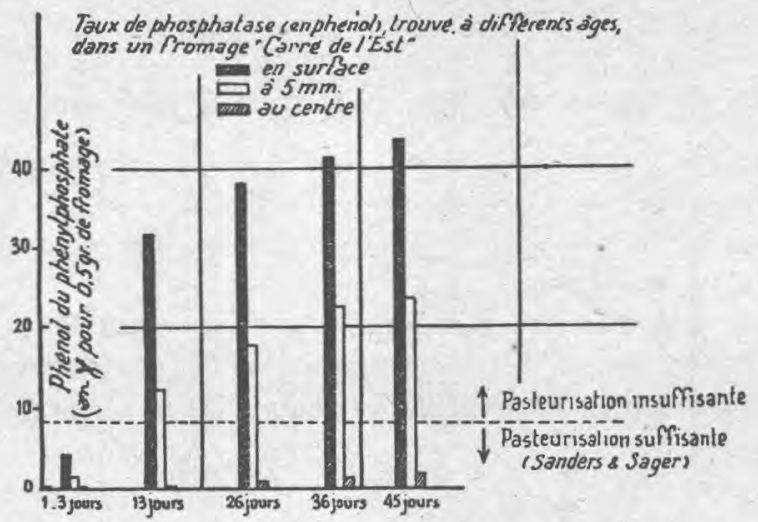

déjà très important après treize jours. Mais, même après quarantecinq jours, il reste encore nul au centre du fromage. A cet endroit, le test conserve sa valeur.

TABLEAU II

\begin{tabular}{|c|c|c|c|c|c|c|}
\hline & $1^{\text {er jour }}$ & $3^{e}$ jour & $13^{e}$ jour & $26^{\mathrm{e}}$ jour & 36 ejour & $45^{\mathrm{e}}$ jour \\
\hline En surface $\ldots \ldots \ldots \ldots$ & 0 & 4 & 30 & 38 & 40,5 & 43 \\
\hline A $5 \mathrm{~mm}$. de la surface. & 0 & 1 & 12 & 18 & 22 & 25 \\
\hline Au centre $\ldots \ldots \ldots \ldots$ & 0 & 0 & 0 & 1 & 1 & 1 \\
\hline
\end{tabular}

Nous tirerons cependant de ces constatations les conclusions suivantes :

1. Le fait que le taux de phosphatase n'a pas varié au centre du fromage, malgré l'achèvement de la maturation et la protéolyse, semble bien prouver que la phosphatase apparue dans la zone intermédiaire provient de la surface. La coloration des essais à blanc, chauffés (Cf. Technique du dosage de Sanders et Sager), permet par ailleurs de séparer l'influence des radicaux phénols des acides aminés libérés au cours de l'affinage de celle du phénol libéré du phénylphosphate par la phosphatase.

2. Il convient d'observer une extrême prudence dans l'interprétation des résultats chaque fois que l'essai est effectué sur un fromage affiné. Même en respectant les précautions indiquées par 
Sanders et SAGer (loc. cit.), dans le but d'éviter l'entraînement de fragments de la croûte dans l'échantillon prélevé au centre, il devient difficile de faire le prélèvement dans la zone de faible épaisseur dépourvue de phosphatase de certains fromages plats. frais.

Il sera toujours préférable d'appliquer le test sur des fromages

\section{Essais effectués sur des fromages à pâte pressée, cuite}

Nous avons émis l'hypothèse que, dans les fromages du type Gruyère, la formation d'une croûte et les soins réguliers apportés à celle-ci, sont peut-être un obstacle à la formation de phosphatase fongique ou microbienne.

Un essai analogue aux précédents, avec des prélèvements effectués dans la croûte, à $2^{\circ}$ et 4 centimètres de profondeur, nous a conduits aux résultats exprimés au tableau III et au graphique III.

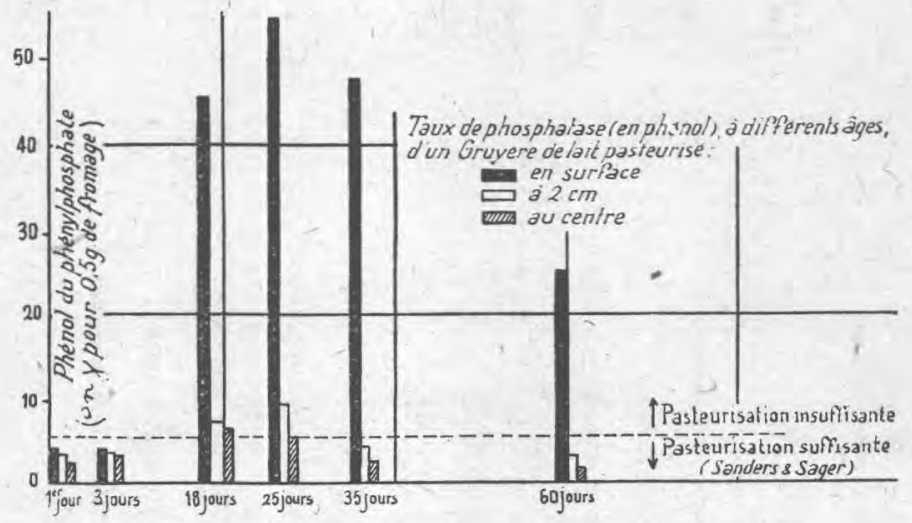

On assiste, dans toute la masse du fromage, à une augmentation du taux de phosphatase qui passe par un maximum pour diminuer ensuite.

\section{TABLEAU III}

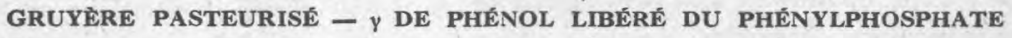

\begin{tabular}{l|c|c|c|c|c|c}
\hline \hline & $\begin{array}{c}1^{\mathrm{er}} \text { jour } \\
\text { sortie de } \\
\text { presse }\end{array}$ & $\begin{array}{c}3^{\mathrm{e}} \text { jour } \\
\text { sortie de } \\
\text { saumure }\end{array}$ & $18^{\mathrm{e}}$ jour & $255^{\mathrm{e}}$ jour & $35^{\mathrm{e}}$ jour & $59^{\mathrm{e}}$ jour \\
\hline Dans la crô̂te ....... & 4 & 4 & 45 & 55 & 46 & 26 \\
A 2 cm. de lacroûte... & 4 & 4 & 7 & 9,5 & 5 & 3,5 \\
A $4 \mathrm{~cm}$. de lacroûte... & 2 & 2 & 7,5 & 6 & 3 & 1 \\
\hline \hline
\end{tabular}


Si l'on se réfère au travail de SANDERS et SAGER (loc. cit.) qui fixent à $6 \gamma$ de phénol libéré pour 0 gr. 5 de fromage la limite au-dessus de laquelle un fromage doit être considéré comme provenant d'un lait insuffisamment pasteurisé, le gruyère soumis à l'essai, à phosphatase négative au début, n'aurait pas résisté à l'épreuve entre le $15^{\mathrm{e}}$ et le $30^{\mathrm{e}}$ jour de sa maturation. Par contre, après un mois, il pouvait à nouveau être considéré comme provenant d'un lait pasteurisé.

Cette disparition de la phosphatase méritait une confirmation : nous l'avons vérifié sur deux petits gruyères provenant d'un lait insuffisamment pasteurisé, prélevés dans une usine qui recherche non pas la pasteurisation effective, mais un simple "chauffage d'arrêt " $\left(75^{\circ}\right)$ lui permettant la conduite normale de sa fabrication.

Les résultats consignés aux tableaux IV et $\mathrm{V}$ ont servi à l'établissement des graphiques correspondants.

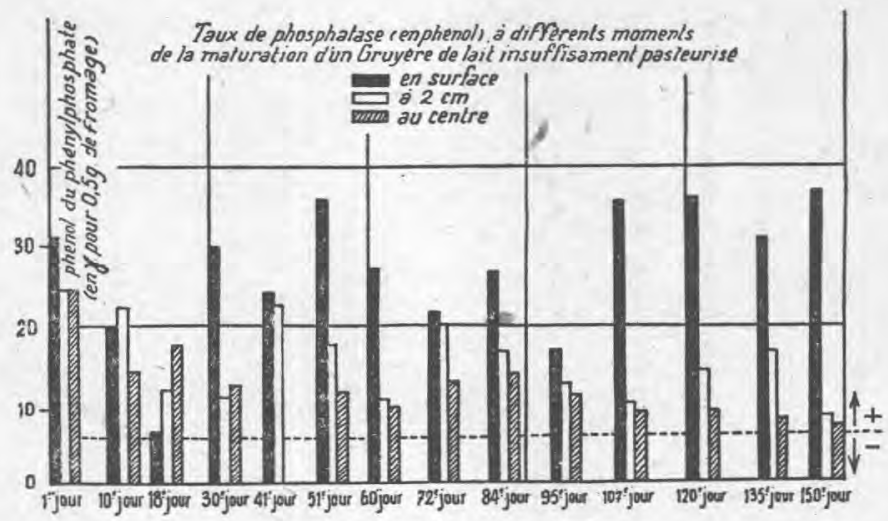

TABLEAU IV

\begin{tabular}{|c|c|c|c|c|c|c|c|c|c|c|c|c|c|c|}
\hline & $\begin{array}{l}\text { Ier jour } \\
\text { sortie de } \\
\text { saumure }\end{array}$ & $\begin{array}{l}10^{\mathrm{e}} \\
\text { jour }\end{array}$ & $\begin{array}{l}18^{e} \\
\text { jour }\end{array}$ & $\begin{array}{l}30^{\mathrm{e}} \\
\text { jour }\end{array}$ & $\begin{array}{l}41^{e} \\
\text { jour }\end{array}$ & $\begin{array}{l}51^{e} \\
\text { jour }\end{array}$ & $\begin{array}{l}60^{e} \\
\text { jour }\end{array}$ & $\begin{array}{l}72^{e} \\
\text { jour }\end{array}$ & $\begin{array}{l}84^{e} \\
\text { jour }\end{array}$ & $\begin{array}{l}95^{\mathrm{e}} \\
\text { jour }\end{array}$ & $\begin{array}{l}107 \mathrm{e} \\
\text { jour }\end{array}$ & $\begin{array}{l}120^{e} \\
\text { jour }\end{array}$ & $\begin{array}{l}135^{e} \\
\text { jour }\end{array}$ & $\begin{array}{l}147^{e} \\
\text { jour }\end{array}$ \\
\hline Croûte ... & 29 & 20 & 6 & 28 & 24 & 35 & 27 & 21 & 26,5 & 17 & 36 & 36 & 31 & 36,5 \\
\hline A $2 \mathrm{~cm}, \ldots$ & 24 & 22 & 13 & 11 & 23 & 18 & 11 & 20 & 17 & 13 & 11 & 13 & 17 & 8 \\
\hline A $4.5 \mathrm{~cm}$. & 24 & 15 & 17 & 13 & 一 & 12 & 10 & 13 & 14,5 & 12 & 9,5 & 9 & 8 & 8 \\
\hline
\end{tabular}

Le taux de phosphatase de la croûte présente des irrégularités : les points bas coïncident avec des prélèvements effectués immédiatement après les soins en cave. A partir du moment où ces soins 
ont cessé, la eroûte étant bien formée, le taux de phosphatase se stabilise. Il convient de noter l'influence du taux de phosphatase de la croûte sur celui de la partie sous-jacente, qui présente, aussi, des irrégularités moins prononcées.

TABLEAU $V$

\begin{tabular}{l|c|c|c|c|c|c|c|c|c|c|c|c}
\hline \hline & $\begin{array}{c}1^{\mathrm{er}} \text { jour } \\
\text { sortie de } \\
\text { saumure. }\end{array}$ & $\begin{array}{c}10^{\mathrm{e}} \\
\text { jour }\end{array}$ & $\begin{array}{c}28^{\mathrm{e}} \\
\text { jour }\end{array}$ & $\begin{array}{c}45^{\mathrm{e}} \\
\text { jour }\end{array}$ & $\begin{array}{c}47^{\mathrm{e}} \\
\text { jour }\end{array}$ & $\begin{array}{c}69^{\mathrm{e}} \\
\text { jour }\end{array}$ & $\begin{array}{c}77^{\mathrm{e}} \\
\text { jour }\end{array}$ & $\begin{array}{c}88^{\mathrm{e}} \\
\text { jour }\end{array}$ & $\begin{array}{c}103^{\mathrm{e}} \\
\text { jour }\end{array}$ & $\begin{array}{c}117^{\mathrm{e}} \\
\text { jour }\end{array}$ & $\begin{array}{c}130^{\mathrm{e}} \\
\text { jour }\end{array}$ & $\begin{array}{c}153^{\mathrm{e}} \\
\text { jour }\end{array}$ \\
\hline Croûte $\ldots$ & 26 & 23 & 12 & 24,5 & 11 & 28 & 23 & 15 & - & 31 & 30 & 25 \\
A 2 cm.... & 11 & 17 & 13 & 9 & 16 & 12,5 & 11 & 8 & 6 & 8,5 & 8,5 & 7,5 \\
A 4-5 cm, . & 13 & 13 & 11 & 6,5 & 7,5 & 8 & 4,5 & 8 & - & 6 & 6 & 5 \\
\hline \hline
\end{tabular}

Mais la chute lente et continue du taux de phosphatase dans le centre du fromage doit surtout retenir notre attention. Le taux de phénol libéré passe, en-effet, pour l'un des fromages de 24 à $8 \gamma$ ct pour l'autre de 13 à $5 \gamma$. Ce dernier fromage (graphique $V$ ), bien qu'insuffisamment pasteurisé présente, après cinq mois de stockage, une réaction de la phosphatase négative (selon SANDERS et SAGER) ou tout au moins douteuse.

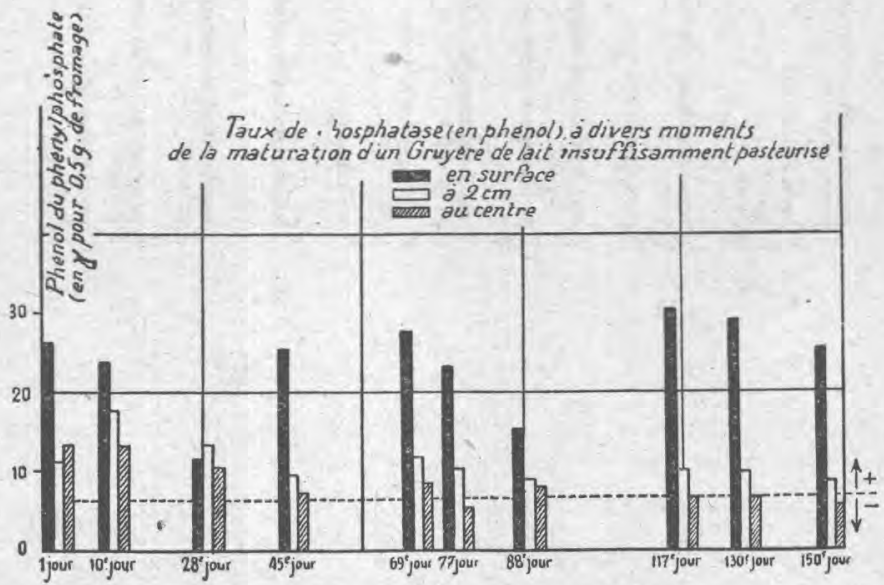

Contrairement à notre attente, le test de la phosphatase se heurte donc dans les fromages du type Gruyère à des obstacles encore plus importants que ceux rencontrés dans les fromages à pâte molle et à croûte moisie. En effet, un fromage provenant d'un lait effectivement pasteurisé peut être jugé comme non pasteurisé dans les premiers mois de sa maturation; mais, par ailleurs, un fromage pro- 
venant d'un lait cru, pauvre en phosphatase, ou d'un lait insuffisamment chauffé peut, après quelques mois de conservation, passer pour un fromage de lait pasteurisé.

\section{Hypothèse sur la disparition de la phosphatase dans le fromage de Gruyère}

Etant donné les phénomènes de dégradation protéolytiquc dont un fromage est le siège au cours de sa maturation, nous avons pensé qu'ils pouvaient intéresser l'apoenzyme de la phosphatase au même titre que les protéines du fromage ; son support protéinique étant disloqué, la diastase perdrait son activité.

Pour vérifier cette hypothèse, nous avons, dans un premier essai, soumis 100 millilitres de lait eru à une digestion à $30^{\circ} \mathrm{C}$. par milligramme de papaīne et de pepsine ; en voici les résultats :

Phénol libéré du phénylphosphate

Avant digestion en $y$

Après 24 heures de digestion par la papaïne ........... 3

Après 24 heures de digestion par la pepsine .......... 1

Mais la conservation du fromage s'effectuant à des températures plus basses, il convenait d'étudier les possibilités d'action des protéinases aux températures normales de maturation.

Des échantillons de 100 millilitres de lait cru ont été additionnés de 0 mgr. 010 de pepsine, de papaïne et de trypsine, et placés à $15^{\circ}$ :

PHÉNOL LIBÉRE PAR LA PHOSPHATASE

\begin{tabular}{|c|c|c|c|c|c|}
\hline & $\begin{array}{l}\text { Au début } \\
\text { de l'essai }\end{array}$ & $\begin{array}{c}\text { Après } \\
72 \text { heures }\end{array}$ & $\begin{array}{c}\text { A près } \\
48 \text { heures }\end{array}$ & $\begin{array}{c}\text { Après } \\
28 \text { heures }\end{array}$ & $\begin{array}{c}\text { Après } \\
19 \text { heures }\end{array}$ \\
\hline $\begin{array}{l}\text { Témoin sans protéinase.. } \\
\text { Lait additionné de pa- }\end{array}$ & 62 & 54 & 53 & 53 & 54 \\
\hline païne $\ldots \ldots \ldots \ldots \ldots$ & 62 & 50 & 45 & 39 & 42 \\
\hline Lait additionné de pepsine & 62 & 58 & 38 & 30 & 18 \\
\hline $\begin{array}{l}\text { Lait additionne de tryp- } \\
\qquad \sin e \ldots \ldots \ldots \ldots \ldots \ldots\end{array}$ & 62 & 54 & 51 & 35 & 18 \\
\hline
\end{tabular}

La diminution du taux de phosphatase est donc encore très nette et très rapide à $15^{\circ}$.

Il n'est done pas exclu de penser que les protéinases du fromage, qu'elles soient d'origine microbienne ou d'origine gastrique (pepsine de la présure restante), jouent un rôle dans la disparition lente de la 
phosphatase au cours dè la maturation du gruyère. Des essais sont en cours pour en préciser les conditions.

\section{Conclusions}

Les essais de dosage de la phosphatase pratiqués régulièrement au cours de la maturation des fromages laissent donc apparaître des difficultés importantes dans l'application de ce test de la pasteurisation des laits de fromagerie.

Dans les fromages à pâte molle, à croûte moisie, les phosphatases microbiennes et fongiques de surface pénètrent dans la masse. En général, elles n'ont pas le temps d'atteindre le centre au cours d'une maturation de durée normale. Cependant, de très grandes précautions doivent être observées dans les prélèvements.

Dans les fromages à pâte pressée, cuite, du type Gruyère, ces phosphatases apparaissent dans toute la masse ; mais, par contre, elles disparaissent ensuite lentement, au point qu'un fromage de lait insuffisamment pasteurisé peut présenter une réaction de phosphatase négative après quelques mois de stockage et passer pour un fromage de lait pasteurisé.

A l'époque où, dans les transactions commerciales, l'épreuve de la phosphatase appliquée aux fromages, se trouve préconisée de plus en plus fréquemment comme test de l'efficacité de la pasteurisation de la matière première, ces quelques points méritent de retenir l'attention et doivent inciter à la plus grande prudence dans la rédaction des conclusions et dans la généralisation trop hâtive de la méthode, qui semble n'avoir actuellement de valeur certaine que pour les fromages frais.

\section{Remerciements :}

Nous adressons nos remerciements à $M$. Gérard, industriel laitier au Tholy et à la Société des Fermiers Réunis qui ont bien voulu mettre leur matériel à notre disposition pour la fabrication des fromages d'essai.

[1] A. Canus. Le sort de la phosphatase dans la conservation des fromages. Communication au $X I I^{\mathrm{e}}$ Congrès International de Laiterie, Stockholm, 1949.

[2] G. P. SAnders et O. S. SAger. Phosphatase test for various dairy products. Journal of Dairy Science, vol. XXX, décembre 1947. 